\title{
A DEDICATED SYNCHROTRON LIGHT SOURCE FOR ULTRAFAST X-RAY SCIENCE*
}

\author{
J. Corlett, S. DeSantis, N. Hartman, P. Heimann, R. Lafever, D. Li, H. Padmore, R. Rimmer, \\ K.E. Robinson, R. Schoenlein, J. Tanabe, S. Wang, and A. Zholents" ${ }^{\#}$ LBNL, Berkeley, CA 94720, \\ USA \\ D. Kairan, Budker INP, 630090, Russia
}

\begin{abstract}
We describe a proposed femtosecond synchrotron radiation $\mathrm{x}$-ray source based on a flat-beam $\mathrm{RF}$ gun and a recirculating superconducting linac that provides beam to an array of undulators and bend magnets. X-ray pulse durations of $<100 \mathrm{fs}$ at a $10 \mathrm{kHz}$ repetition rate are obtained by a combination of electron pulse compression, transverse temporal correlation of the electrons, and x-ray pulse compression.
\end{abstract}

\section{INTRODUCTION}

Ultra-fast science is an important new research frontier that is driving the development of novel sources for the generation of extremely short x-ray and electron pulses. Recent advances in femtosecond lasers have stimulated development of femtosecond $\mathrm{x}$-ray sources that allow the study of matter at the time scale shorter than period of oscillations of atoms in molecules, $\sim 100 \mathrm{fs}$ (see, [1] and references therein). In this paper, we propose a high peak and average flux source of femtosecond x-ray pulses. This source would be optimized for pump-probe experiments utilizing femtosecond pump laser pulses and femtosecond $\mathrm{x}$-ray probe pulses. As this is to be a dedicated facility, we chose the repetition rate, $10 \mathrm{kHz}$, to match the relaxation times, acquisition rates and femtosecond pump lasers necessary to investigate ultra fast phenomena. The design parameters of the machine are rather modest: a beam energy of $2.5 \mathrm{GeV}$, bunch charge of $\sim 1 \mathrm{nC}$ (average beam current of $10 \mu \mathrm{A}$ ), an initial electron bunch length of $10 \mathrm{ps}$ that later is compressed to $1 \mathrm{ps}$ at $2.5 \mathrm{GeV}$. These parameters will result in the $\mathrm{x}$-ray flux of undulator radiation of $\sim 5 \times 10^{10}$ photons per $0.1 \%$ bandwidth at photon energy of $8 \mathrm{keV}$.

The machine consists of an injector, a linear preaccelerator, a main linear accelerator, magnetic arcs, and a production beam section (undulator farm). The machine layout is shown in Figure 1. Electrons, produced in an RF photocathode gun, are accelerated to $10 \mathrm{MeV}$ and further accelerated in a pre-accelerator to $120 \mathrm{MeV}$. They are then injected into a recirculating linear accelerator, where their final energy of $\sim 2.5 \mathrm{GeV}$ is achieved after four passes. After the fourth pass, an arc transports the electrons into the undulator farm where they radiate $\mathrm{x}$-rays in the undulators and dipole magnets. There are seven two-meter long straight sections for undulators in the production section. There are also six $2 \mathrm{~T}$ dipole magnets located between the undulators each with a $60 \mathrm{mrad}$ arc angle. After passing through the undulator farm, the electrons are decelerated in the same linacs to $\sim 10 \mathrm{MeV}$ and turned into the beam dump. This deceleration reduces the radiation hazard of the beam dump.

All arcs are designed to be achromatic and, except the fourth, are isochronous. The fourth arc compresses the electron bunch from $\sim 10 \mathrm{ps}$ to $\sim 1 \mathrm{ps}$. After the beam passes the undulator farm, it is stretched back to $\sim 10 \mathrm{ps}$ in the return arc. The arcs are each designed with three identical $120^{\circ}$ betatron phase advance cells and the

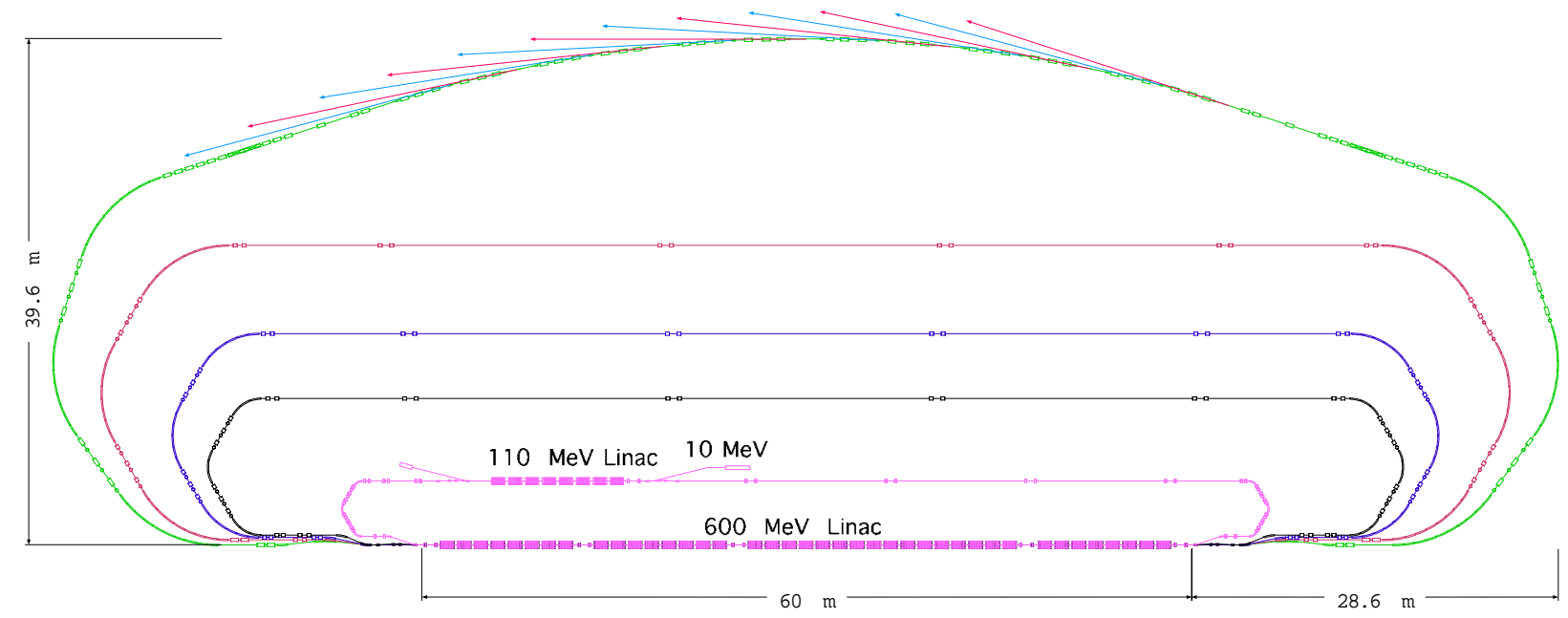

Figure 1. Configuration of the femtosecond synchrotron radiation x-ray source

\footnotetext{
*This work was supported by the U.S.Department of Energy under
} Contract No. DE-AC03-76SF00098. \# AAZholents@1bl.gov 
flexibility to establish the betatron phase advance to allow matching of the beam on successive passes through the recirculating linac. The last lattice cell of each arc is modified to match to the straight section. The straight sections provide successive phase adjustment. The separation of the different energy beams into their respective arcs is accomplished by a beam spreader lattice section. The lattice of the undulator farm consists of six identical cells and two matching sections on both sides.

At the beginning and end of the undulator farm, we use two RF structures that operate with the first dipole mode $\mathrm{E}_{110}$ to provide a time dependent transverse kick to the electron beam that the second structure cancels. The electrons perform free betatron oscillations after the first kick, with amplitude 10-20 times larger than the vertical beam size. These oscillations allow the emitted x-ray pulse to be compressed [2].

The phase advance for vertical betatron oscillations between the center of RF structure and the center of the $n t h$ undulator is $n \pi$. The vertical betatron phase advance between the center of the undulator and the center of the adjacent $2 \mathrm{~T}$ dipole magnet is $\pi / 2$. All undulators and $2 \mathrm{~T}$ magnets are sources of $\mathrm{x}$-rays with spatial-time correlation: electron displacements are maximum in the dipoles and angle variation is maximum in the undulators.

\section{FLAT E-BEAM PRODUCTION}

The approach for producing a short $\mathrm{X}$-ray pulse relies on the availability of an electron beam with a flat aspect ratio so that one transverse dimension (vertical) is small when compared to the radiation diffraction limit. Derbenev conceived a method of producing circular beams from a flat-beam cross-section first applied to storage rings [3] then later, an inverse transformation from a cathode [4]. An emittance ratio of order $10^{2}$ may be achieved by this procedure, with a normalized emittance of $\left(\varepsilon_{\mathrm{x}} \times \varepsilon_{\mathrm{y}}\right)^{0.5}$ - $10^{-6} \mathrm{~m}-\mathrm{rad} / \mathrm{nC}$ at the cathode.

The principal has been successfully demonstrated at Fermilab [5]. An emittance ratio of 50:1 was measured on a $1 \mathrm{nC}$ bunch with measured horizontal and vertical emittances of $\varepsilon_{x}=0.9 \times 10^{-6} \mathrm{~m}-\mathrm{rad}, \varepsilon_{\mathrm{y}}=45 \times 10^{-6} \mathrm{~m}-\mathrm{rad}$. Consequently, we may reasonably expect to achieve an emittance ratio of 50:1 for $1 \mathrm{nC}$ charge with $\varepsilon_{y} \bullet 0.4 \times 10^{-6}$ $\mathrm{m}$-rad and $\varepsilon_{x} \cdot 20 \times 10^{-6} \mathrm{~m}$-rad based the results of this proof-of-principle experiment.

\section{ACCELERATOR SYSTEMS}

\section{$R F$ gun}

Conventional photocathode RF guns employ a halflength pillbox cell for the gun cavity followed by one or more normal cells for rapid acceleration. These have been shown to give good emittance at high gradient when operating at a low duty factor. Typical RF guns have accelerating fields of $\sim 50 \mathrm{MV} / \mathrm{m}$, and low repetition rates of the order of $10 \mathrm{~Hz}$. This is in contrast to our required rate of $10 \mathrm{kHz}$ or greater. For $\mathrm{CW}$, or high duty-factor operation, thermal limitations may prevent such a design from operating at a sufficiently high gradient. A re-entrant shape for the gun cell with good cooling may solve this problem.

We have produced a conceptual design with an optimized cavity geometry that allows cooling of the cavity surfaces, and operation at high gradient and high repetition rate. The first cell is modified by the inclusion of a large nose cone, on which the photocathode is mounted. This nose cone increases surface area to reduce deposited power density, and enhances the field at the cathode.

Parameters have been calculated using the URMEL code for various repetition rates and cavity frequencies. The maximum power density dissipated by RF currents in the cavity walls is limited to $100 \mathrm{~W} / \mathrm{cm}^{2}$ to allow for adequate cooling by water channels, and acceptable stress levels in the copper. From ANSYS modeling of the cavity geometry, an acceptable accelerating gradient of $40 \mathrm{MV} / \mathrm{m}$ or greater is achieved even at repetition rates of $100 \mathrm{kHz}$ with the $1.3 \mathrm{GHz}$ cavity in contrast to an equivalent pillbox geometry that is limited to $\sim 25 \mathrm{MV} / \mathrm{m}$. The maximum temperature rise is to $68^{\circ} \mathrm{C}$, and the thermal stresses are suitable for a copper structure.

\section{RF systems and linacs}

Our linac design is based on the superconducting RF technology developed for the TESLA project [6]. TESLA RF cavity parameters are well suited to our requirements.

Each cryomodule is $12 \mathrm{~m}$ long, and we require four cryomodules to achieve an energy gain of $>600 \mathrm{MeV} /$ pass corresponding to an accelerating gradient of $\sim 20 \mathrm{MV} / \mathrm{m}$. Operating at the TESLA gradient of $23 \mathrm{MV} / \mathrm{m}$ would allow a final beam energy of $\sim 3 \mathrm{GeV}$, but the lower gradient provides a design margin that simplifies the lattice, and reduces construction and operating costs. An additional identical cryomodule is used for the injector linac.

The superconducting cavities have a high quality factor, resulting in long filling times. We must operate the linac RF system in continuous (CW) mode because of the high pulse repetition rate. This increases the power requirements of the system - both in rating of the RF drive power system, and in the cryogenic system capacity needed for resistive losses in the cavities. Table 1 summarizes the RF system requirements. The cavities are not heavily beam loaded with a required beam current of

Table 1. Femtosecond x-ray source RF requirements

\begin{tabular}{|l|l|}
\hline Energy gain per pass & $600 \mathrm{MeV}$ \\
\hline $\mathrm{E}_{\text {accelerating }}$ & $20 \mathrm{MV} / \mathrm{m}$ \\
\hline Operating mode & $\mathrm{CW}$ \\
\hline Dynamic power losses per cavity & $42 \mathrm{~W}$ \\
\hline Average beam current & $0.04 \mathrm{~mA}$ \\
\hline Beam power requirement & $25 \mathrm{~kW}$ \\
\hline $\mathrm{Q}_{\text {mbaaded }}$ & $1 \times 10^{10}$ \\
\hline $\mathrm{Q}_{\text {extemal }}$ & $6.5 \times 10^{6}$ \\
\hline Cavity resonance bandwidth & $200 \mathrm{~Hz}$ \\
\hline Cavity coupling factor & $1.54 \times 10^{3}$ \\
\hline RF losses per cryomodule & $336 \mathrm{~W}$ \\
\hline RF power requirement per cryomodule & $128 \mathrm{~kW}$ \\
\hline Linac RF klystron rating & $1 \mathrm{MW}$ \\
\hline
\end{tabular}


only $0.04 \mathrm{~mA}$. The total electron beam power requirement is $25 \mathrm{~kW}$ for main linac and injector. The power dissipated in the cavity walls is $42 \mathrm{~W}$ per cavity and represents only $0.259 \%$ of the incident power. The incident power required to produce $20 \mathrm{MV} / \mathrm{m}$ is $16 \mathrm{~kW}$ per cavity, or a total requirement of $665 \mathrm{~kW}$ for the main linac and injector cryomodules and electron beam power.

\section{Cryogenics system}

In addition to the dynamic RF losses, the cryogenic system must remove persistent or static losses due to conduction from surroundings outside the cold mass. Static losses for the TESLA cryomodules operating at $2^{\circ} \mathrm{K}$ are $0.1 \mathrm{~W} / \mathrm{m}$ or $1.2 \mathrm{~W}$ in for each of the 8-cavity cryomodules [6] (we assume $6 \mathrm{~W}$ static losses, which is small compared to our total dynamic losses of $1.68 \mathrm{~kW}$ ).

BSC theory describes superconducting surface impedance. An exponential factor dominates the temperature dependence, and losses increase by a factor of 41 when operating at $4^{\circ} \mathrm{K}$ compared to operation at $2^{\circ} \mathrm{K}$ [7]. Consequently, there appears to be no advantage to operating at higher temperature.

We specify a $50 \%$ design margin on the $20 \mathrm{MV} / \mathrm{m}$ cryogenic loads so that the cryogenic system is then specified at $2^{\circ} \mathrm{K}$ for a $2.5 \mathrm{~kW}$ load and an AC power requirement of $2 \mathrm{MW}$. This is a significant difference in comparison to the TESLA cyrogenic systems that are designed for $2 \mathrm{~K}$ loads of only $9 \mathrm{~W}$

\section{X-RAY PULSE COMPRESSION}

$\mathrm{X}$-ray pulse compression requires spatial-time correlation in the $\mathrm{X}$-ray pulses to permit a final pulse length of less than $100 \mathrm{fs}$. Asymmetrically cut crystals are used as optical elements for X-ray pulse compression $[2,8]$. Because of the different incident and diffractive angles, they produce a variable path length across the $\mathrm{x}$ ray beam. After the collection optics, two asymmetrically cut crystals can act as monochromator and pulse compressor for the $\mathrm{x}$-rays. It is necessary to add a second rotation of the crystals in addition to the Bragg angle adjustment. The crystals are rotated about the normal to the Bragg planes, $\phi$, which varies the crystal asymmetry in the diffraction plane. [9]. It is possible to tune the monochromator photon energy while keeping the $\mathrm{x}$-ray pulse compression fixed. Kirkpatrick-Baez mirrors are used to refocus the x-rays onto the samples after the crystal.

\section{SUMMARY}

We are continuing further investigation of this $\sim 50$ femtosecond x-ray synchrotron radiation source [10]. The approach for much of the source builds upon demonstrated and developed designs such as TESLA [6] and the FNAL A0 [5] systems, but is complementary to other work being proposed in this area [11]. However, the demands necessary to achieve a dedicated scientific source require significant departures from these existing designs. The high repetition rate requires $\mathrm{CW}$ operation of the linacs with increased demands on the RF distribution and cryogenic systems. This repetition rate also impacts the design of the RF gun that likewise requires careful development to achieve the necessary operating characteristics. The approach for meeting these development challenges is straightforward. With this ultra fast synchrotron radiation $\mathrm{x}$-ray source it will be possible to directly measure the motion of molecules and atoms in very fast dynamic systems as well as provide sufficient flux for weak scattering systems. This source will also be capable of investigating disordered systems and liquids and represents a major step forward in ultrafast dynamics research.

\section{REFERENCES}

[1] R. W. Schoenlein, et. al., Generation of Femtosecond $X$-ray Pulses via Laser-Electron Beam Interaction, Appl. Phys. B, 1-10, 2000.

[2] A. Zholents, P. Heimann, M. Zolotorev, J. Byrd, "Generation of subpicosecond $\mathrm{x}$-ray pulses using RF orbit deflection", NIM A 425 (1999)385-389.

[3] Y. Derbenev, "Adapting Optics for High Energy Electron Cooling", University of Michigan Report No. UM-HE-98-04, 1998.

[4] R. Brinkmann, Y. Derbenev, K. Flottmann, "A FlatBeam Electron Source for Linear Colliders", DESY Report No. TESLA 99-09, 1999.

[5] D. Edwards et al, "The Flat Beam Experiment at the FNAL Photoinjector", Proc. XXth International Linac Conference, Monterey, 2000 (to be published).

[6] TESLA Technical Design Report, DESY 2001-011, March 2001.

[7] H. Padamse, J. Knobloch, T. Hays, "RF Superconductivity for Accelerators", John Wiley \& Sons Inc, ISBN 0-471-15432-6, 1998.

[8] T. Matsushita and H. Hashizume, Handbook of Synchrotron Radiation V.1, ed. E.E. Koch, p.261, North-Holland, Amsterdam (1993).

[9] R.K. Smither, P.B. Fernandez, Nuclear Instrum. And Methods A 347, 313 (1994).

[10] For additional details on this source see, J. Corlett, et al., LBNL-48171, June 2001; and A. Zholents, On the possibility of a femtosecond $x$-ray source based on a recirculating linac, CBP TECH-Note-219, 14 Nov. 2000.

[11] See for example: I. Ben-Zvi, et al., Photoinjected Energy Recovery Linac Upgrade for the National Synchrotron Light Source, these proceedings; D.A. Kayran, et al., MARS $-A$ Project of the Diffraction Limited Fourth Generation X-ray Source, Proc. 1998 Asian Particle Accelerator Conf.; and I. V. Bazarov, et al., The Energy Recovery Linac (ERL) as a Driver for X-ray Producing Insertion Devices, these proceedings, among others. 